\title{
How to perform surgical planning for hemodialysis access? Routine preoperative doppler ultrasound mapping
}

\author{
Nurullah Doğan, Ömer Fatih $\operatorname{Nas}^{2} \odot$ \\ ${ }^{1}$ Department of Radiology, Doruk Medical Center, Bursa, Turkey \\ ${ }^{2}$ Department of Radiology, Uludağ University School of Medicine, Bursa, Turkey
}

\begin{abstract}
Objectives: The aim of the study was to evaluate the effect of routine preoperative Doppler ultrasound mapping on surgical planning in patients having hemodialysis fistulas.

Methods: Patients with arteriovenous fistulas (AVF) or arteriovenous grafts (AVG) were retrospectively reviewed between June 2010 and July 2017. Physical findings, preoperative Doppler ultrasound and postoperative findings of 1924 patients were reviewed.

Results: Fistula maturation rate was $82 \%$. The average duration of patency of the first fistula was 19 months (3-38 months) and the duration for a previous fistula operation history was 11 months (1-14 months). Patency rate after 6 months was $78 \%$. Mean maturation times for AVF and AVG were 70 and 28 days, respectively. Doppler ultrasound findings affected operation plans of surgeons in 227 of $908(25 \%)$ first time fistula operation patients, and in 569 of $1016(56 \%)$ patients with previous history of fistula surgery. Review of two groups revealed that Doppler ultrasound affected 41\% (796/1924) of fistula planning of surgery for hemodialysis.

Conclusions: We suggest that preoperative Doppler ultrasound should be routinely performed in patients undergoing fistulation for hemodialysis. Furthermore, a vascular radiologist and a vascular surgeon should decide the surgical plan together.

Keywords: arteriovenous fistulas, arteriovenous grafts, Doppler ultrasound, hemodialysis
\end{abstract}

A $\mathrm{n}$ arteriovenous fistula (AVF) has become the gold standard modality for hemodialysis because of its long patency, good durability and low infection risk [1]. It has been suggested that Doppler ultrasound (DUS) should be performed after clinical examination and before AVF surgery. By this way, AVF failure rate can be reduced and negative surgeries can be avoided $[2,3]$.

At our institution DUS is routinely performed before hemodialysis fistulation in all patients. In this study we aimed to evaluate the effect of routine preoperative DUS mapping on surgical planning.

\section{METHODS}

\section{Data Collection}

Patients having AVF or arteriovenous grafts (AVG) were retrospectively reviewed between June 2010 and July 2017 in our hospital. All of patients were examined before hemodialysis fistulation by one of two experienced vascular surgeons. Consultations by a cardiologist, pulmonologist, anesthesiologist, and/or studies accepting Doppler ultrasound were also included. The surgeon noted the first preoperative plan into patient folders after the physical examination. The 
vascular radiologist and vascular surgeon made their decision on the final preoperative plan according to DUS examination. In some circumstances, the other vascular surgeon also evaluated the patient and the result was stated as a "council decision" in the folder. Initial and final plans in the patient folder were compared. "Doppler ultrasound affected surgery" group was composed of patients whose surgical plans were made following Doppler ultrasound due to reasons such as obesity, edema, or multiple surgical operations.

Patients with inadequate data for the study or who were evaluated with radiological modalities like venography (except Doppler ultrasound) were excluded from the study.

\section{Preoperative Doppler Ultrasound}

Preoperative DUS was performed to all patients prior to fistula surgery for hemodialysis in our hospital.

Upper extremity DUS examinations were made with a GE LOGIQ P7 ultrasound system having 6-12 $\mathrm{MHz}$ probe, in a silent, adjusted heat and light level, and comfortable room. The patient sat against the radiologist with arms protracted forward in a comfortable position.

Arterial examination included longitudinal and transvers scanning of subclavian, axillary, brachial, ulnar and radial arteries. Morphologic parameters such as tracks of the vessels, diameter, wall thickness, changes in the vessel wall, obstructive vessel lesions, and hemodynamic changes were evaluated (Fig. 1).

Venous examination included longitudinal and transverse scanning of superficial and deep veins of the arm (between wrist and distal of the subclavian vein). Parameters evaluated were venous wall thickness, wall structure, flexibility, diameter, track, existence of collateral vessel, and deepness. In case of thin veins, a tourniquet was applied to the brachial region, and this was stated at the report (Figs. 2 and 3). In addition, narrowing of central veins was prevented by using Valsalva maneuver during spectral sampling from the axillary and subclavian levels. If the spectral sampling results were suspicious, venography was made.

\section{Preoperative Planning}

Snuff box AVF, radiocephalic AVF (low, medium, high), brachiocephalic AVF, mobilized basilic vein AVF, ulnabasilic AVF, radiobasilic AVG, brachiocephalic AVG, brachiobasilic AVG, brachiobrachial AVG and brachioaxillary AVG for hemodialysis is made in our unit. The aim was to carry out AVF to the non-dominant arm and the distal part of the arm. If it wasn't suitable for AVF, the dominant arm was used. AVG was performed if no suitable vessel was present for AVF. Patients with history of several fistula operations undergone hybrid fistulas.

The ultimate goal was an artery wider than $1.6 \mathrm{~mm}$ and flow greater than $50 \mathrm{ml} / \mathrm{min}$ (Fig. 1). Veins with thin and smooth walls, anechoic lumen, and the ones which were completely compressible were accepted as normal (Figs 2 and 3). Aimed criteria for veins were a diameter wider than $1.6 \mathrm{~mm}$, depth for the skin less than $1 \mathrm{~cm}$, normal structure vein with an $8-10 \mathrm{~cm}$ straight section.

\section{Statistical Analysis}

Data obtained from files of patients were evaluated from aspects of planning, after physical examinations, and the last planning was done after DUS and fistula maturations. Patients were separated into two groups as ones that were operated for the first time and others that were operated previously. Each group was also analyzed for similarities and differences after physical examination planning data and DUS planning data. The effect of DUS on the preferred surgical plan was investigated.

\section{RESULTS}

A total of 2,133 fistula operations for hemodialysis were made in our hospital between June 2010 and July

\section{Table 1. Demographic parameters of patients}

\begin{tabular}{lc}
\hline Age (year) & 14-82 (mean:58) \\
\hline Sex & \\
\multicolumn{1}{c}{ Male } & 1.022 \\
\multicolumn{1}{c}{ Female } & 902 \\
Hypertension & 543 \\
Diabetes mellitus & 432 \\
Cardiovascular diseases & 522 \\
Smoking & 652 \\
\hline
\end{tabular}


Table 2. Distribution of fistula types

\begin{tabular}{lccc}
\hline Type of Fistula & First Time Fistula & Multipl Time Fistula & Total \\
\hline Snuff box AVF & 47 & 3 & 50 \\
Radiocephalic AVF & 468 & 240 & 708 \\
Brachiocephalic AVF & 295 & 423 & 718 \\
Mobilized basilic vein AVF & 11 & 24 & 35 \\
\hline Ulnar-basilic AVF & 5 & 12 & 17 \\
Radiobasilic AVG & 5 & 5 & 10 \\
Brachiocephalic AVG & 11 & 45 & 56 \\
Brachiobasilic AVG & 61 & 224 & 285 \\
\hline Brachioaxillar AVG & 5 & 40 & 45 \\
Total & 908 & 1.016 & 1.924 \\
\hline AVF Artio
\end{tabular}

$\mathrm{AVF}=$ Arteriovenous fistula, $\mathrm{AVG}=$ : arteriovenous grafts

2017. Demographic data of the patients are shown in Table 1. Two hundred nine patients who did not meet inclusion criteria were excluded. Distribution of fistula types based on the first time operation and previous fistula operation history are shown in Table 2.

Fistula maturation rate was $82 \%$. The average time patency for the first fistula was 19 months (3-38), and the time of previous fistula operation history was 11 months (1-14). The rate of completeness after 6 months was $78 \%$. The mean maturation time was 70 days for AVF and 28 days for prosthetic AVG in the study group.

DUS affected operation plans of the surgeon in
$227(25 \%)$ of the 908 first time fistula operation patients, and $569(56 \%)$ of the 1016 patients with previous history of fistula surgery (Fig. 3). The effect of Doppler ultrasound on the planning of the fistula surgery for hemodialysis was $41 \%$ (796/1924) when the average of two groups was investigated.

\section{DISCUSSION}

Success of AVF procedure affects hemodialysis directly and may have a significant impact on patients. Traditionally, surgeons decide the type of AVF accord-

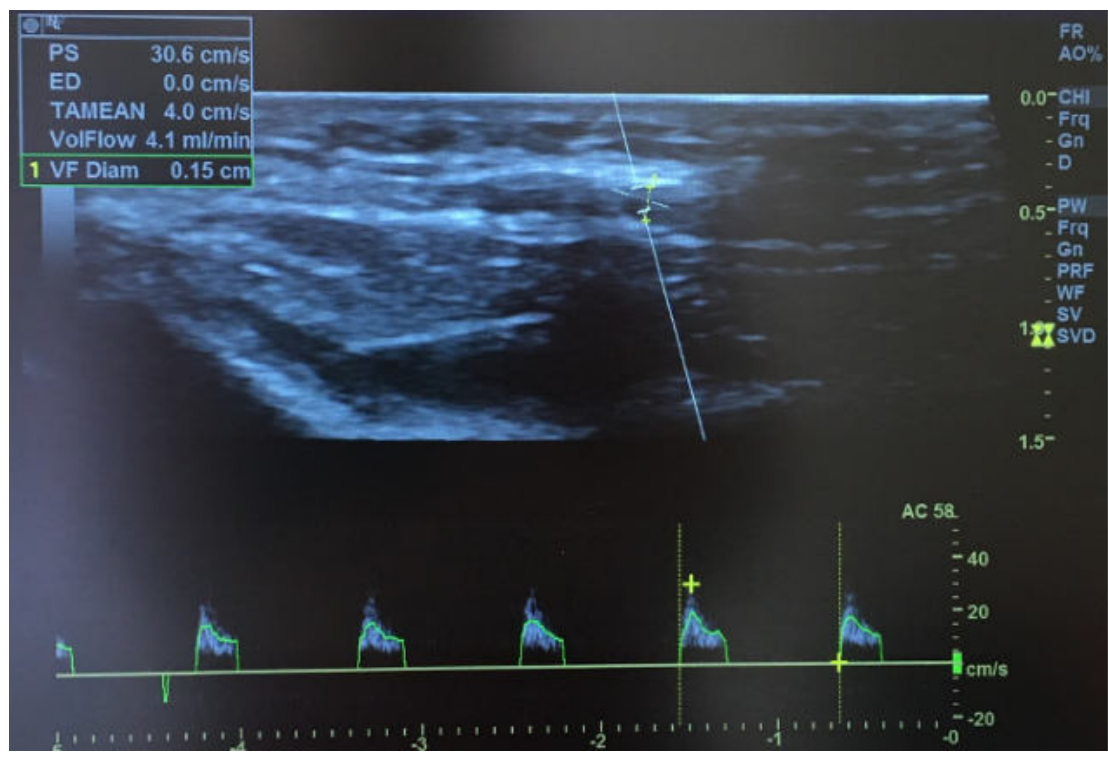

Fig. 1. Extensive calcific atherosclerotic changes are observed in the radial artery wall. Diameter of radial artery is $1.5 \mathrm{~mm}$, flow velocity $4.1 \mathrm{ml} / \mathrm{min}$, flow pattern monophasic. 


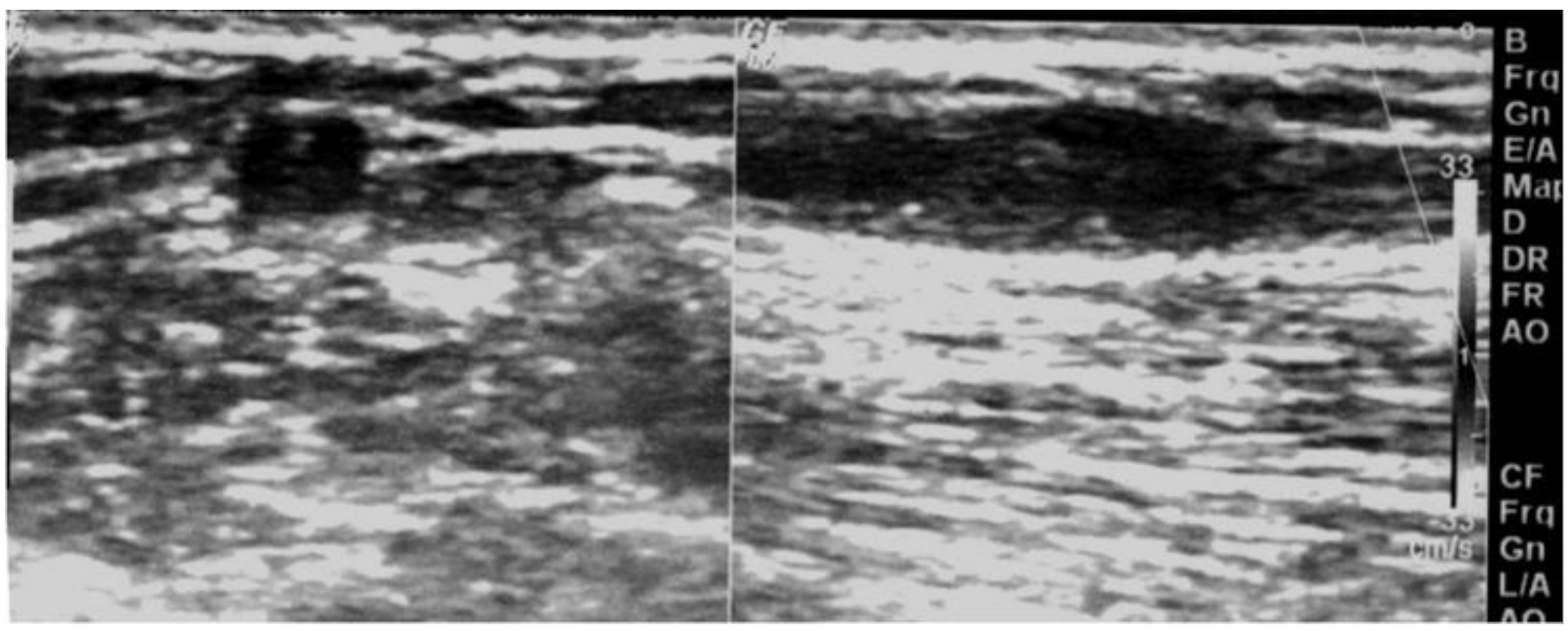

Fig. 2. Axial and sagittal views of the subacute period thrombus in the cephalic vein. Recently, the patient has a history of intracath insertion from this localization.

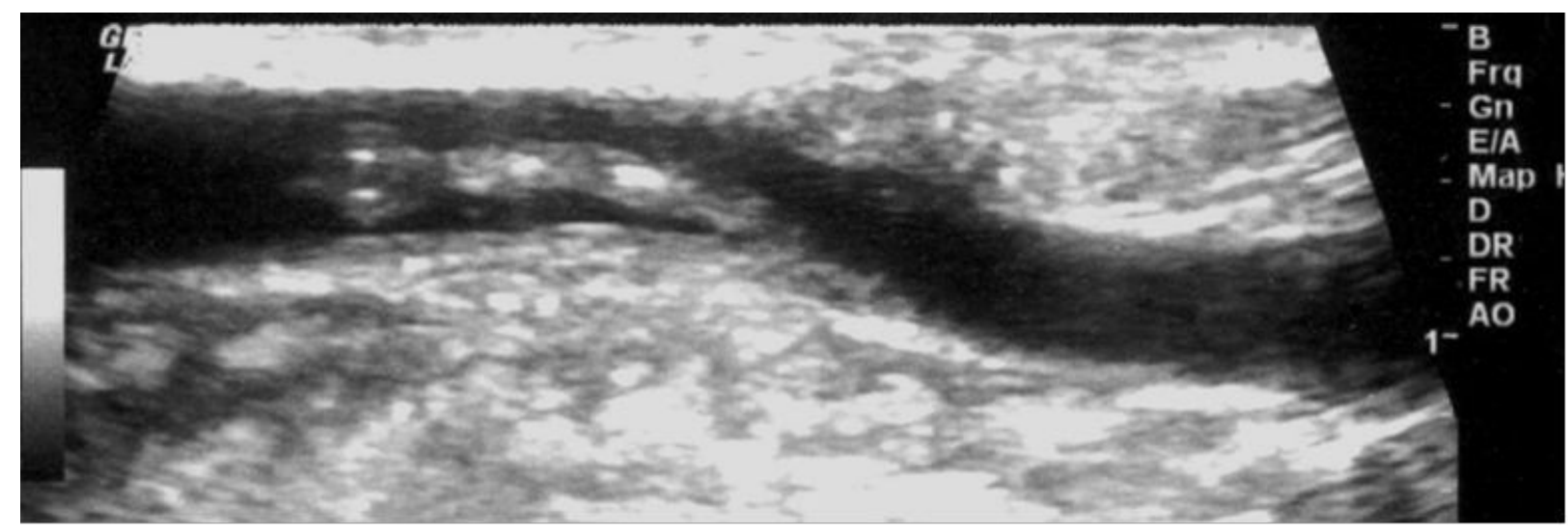

Fig. 3. Axial views of the cronic period thrombus in the cephalic vein.

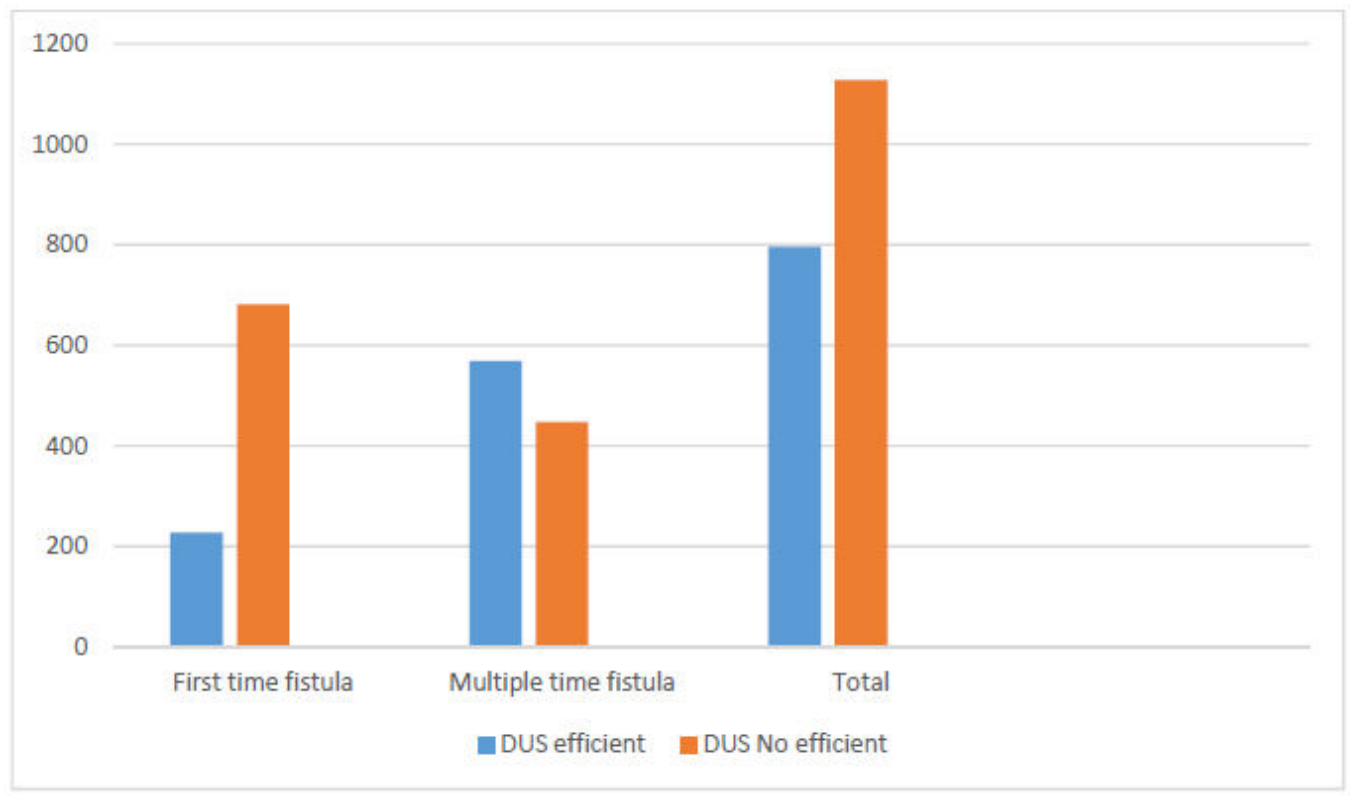

Fig. 4. The effect of Doppler ultrasound on the planning of the fistula surgery for hemodialysis. DUS = Doppler ultrasound 
ing to clinical examination. However, physical examination may not be adequate to decide the AVF type due to conditions of patients like previous fistula surgery, medical comorbidities, etc. This necessitates need of additional examination methods to identify suitable veins [4].

Recently, interest on advantages of ultrasound has increased. It is a noninvasive, safe, and effective method and duplex scanning helps surgeons to improve AVF maturation rates by establishing morphologic and functional parameters or characteristics of vessels [3]. Disadvantages of routine preoperative DUS for hemodialysis fistula are inexperienced vascular radiologists and increased burden on the radiology unit.

Guidelines of the Kidney Diseases Outcomes Quality Initiative recommend routine ultrasound for mapping in all AVF patients while acknowledging the track of Level 1 supportive evidence [5]. Disease Outcomes Quality Initiative guidelines and European Best Practice Guidelines suggest routine use of preoperative ultrasound examination based on level 2 evidence [6]. However, there is no standard accepted DUS mapping routine [7].

It has been stated in a recent meta-analysis study that clinical examination is not sufficient alone for AVF planning and preoperative DUS decreases rates of negative exploration and early AVF failure [4]. In another recently published study, it was shown that the maturation rate was $77.3 \%$ for Doppler ultrasound and $56.8 \%(\mathrm{p}=0.008)$ for non-Doppler ultrasound when patients evaluated by physical examination and by DUS compared [8]. In our study, the maturation rate was $82 \%$ and the 6 month completeness rate was $78 \%$.

Silva et al. [9] postulated in their study that routine preoperative DUS scanning increased the rate of native AVF in their practice from $14 \%$ to $63 \%$. Ferring et al. [10] described that routine preoperative DUS scanning is superior to the use of DUS for the selected patients. Smith et al. [11] reported in their study, with 39 patients, that routine preoperative DUS for fistula operation for hemodialysis caused a $30 \%$ change planning of surgery. This ratio was $41 \%$ in our study. On the other hand, this ratio was $25 \%$ for patients undergoing fistula operation for the first time and a $56 \%$ higher rate was found in patients with previous history of fistula operation.

\section{Limitations}

Limitations of our study was being a single centered study, where one vascular radiologist and two vascular surgeons having experience for working together. We suggest that further studies in different centers will be helpful.

\section{CONCLUSION}

\section{CONCLUSION}

We conclude that performing DUS routinely in the preoperative period is important especially in patients with previous fistula operation history. We recommend that patients fistulated for hemodialysis, should be subjected to preoperative DUS a vascular radiologist and vascular surgeon should decide surgical planning together.

\section{Conflict of interest}

The authors disclosed no conflict of interest during the preparation or publication of this manuscript.

\section{Financing}

The authors disclosed that they did not receive any grant during conduction or writing of this study.

\section{REFERENCES}

1. Bylsma, LC, Gage SM, Reichert H, Dahl SLM, Lawson LH. Arteriovenous fistulae for hemodialysis: a systematic review and meta-analysis of efficacy and safety outcomes. Eur J Vasc Endovasc Surg 2017;54:513-22.

2. Georgiadis GS, Charalampidis DG, Argyriou C, Georgakarakos EI, Lazarides MK. The necessity for routine pre-operative ultrasound mapping before arteriovenous fistula creation: a meta-analysis. Eur J Vasc Endovasc Surg 2015;49:600-5.

3. Zamboli P, Fiorini F, D'Amelio A, Fatuzzu P, Granata A. Color Doppler ultrasound and arteriovenous fistulas for hemodialysis. J Ultrasound 2014;17:253-63.

4. Wong CS, McNicholas N, Healy D, Clarke-Moloney M, Coffey JC, Grace PA, et al. A systematic review of preoperative duplex ultrasonography and arteriovenous fistula formation. J Vasc Surg 2013;57:1129-33.

5. National Kidney Foundation Kidney Disease Outcomes Quality Initiative: 2006 update vascular access. Guideline 2: selection and placement of hemodialysis access. Am J Kidney Dis 2006;48:192-200.

6. Tordoir J, Canaud B, Haage P, Konner K, Basci A, Fouque D, et al. EBPG on vascular access. Nephrol Dial Transplant 
2007;22:88-117.

7. Patel ST, Hughes J, Mills JL. Failure of arteriovenous fistula maturation: an unintended consequence of exceeding Dialysis Outcome Quality Initiative guidelines for hemodialysis access. J Vasc Surg 2003;38:439-45.

8. Mat Said N, Musa KI, Mohamed Daud MA, Haron J. The combination of sonography and physical examination improves the patency and suitability of hemodialysis arteriovenous fistula in vascular access. Malays J Med Sci 2016;23:26-32.

9. Silva MB Jr, Hobson RW II, Pappas PJ, Araki CT, Goldberg
$\mathrm{MC}$, Gwertzman, et al. A strategy for increasing the use of autogenous hemodialysis access procedures: impact of perioperative noninvasive evaluation. J Vasc Surg 1998;27:302-7.

10. Ferring M, Claridge M, Smith SA, Wilmink T. Routine preoperative vascular ultrasound improves patency and use of arteriovenous fistulas for hemodialysis: a randomized trial. Clin J Am Soc Nephrol 2010;5:2236-44.

11. Smith GE, Samuel N, Khan J, Johnson BF, Chetter IC. Targeted duplex ultrasound in a one-stop dialysis vascular access assessment clinic. Ann Vasc Surg 2011;25:1099-103. 